\title{
QUASAR MICROLENSING BY CLUSTER DARK MATTER
}

\section{A $2 d F+M A C H O$ Project}

\author{
M. A. WALKER AND P. M. IRELAND \\ Research Centre for Theoretical Astrophysics, A28 \\ School of Physics, University of Sydney, NSW 2006, Australia
}

\section{Introduction}

Following Paczyński's (1986) suggestion, many examples of gravitational lensing by Galactic objects have been recorded as a consequence of extensive photometric monitoring programs (e.g. Udalski et al. 1994). Now that such experiments have demonstrated the capability to discover microlensing events against large numbers of target sources, it behooves us to consider whether these techniques can be usefully applied elsewhere.

Moving beyond the Galactic realm, we can identify clusters of galaxies as a distinct environment where we might investigate the nature of the dark matter which is dynamically inferred. In this case the photometric targets could not sensibly be stellar; but quasi-stellar objects (quasars) are sufficiently luminous that one can obtain good photometry even of very distant sources. It is therefore worth exploring the possibility that one could detect compact dark matter in galaxy clusters by looking for microlensing events on background quasars; detailed consideration of this idea can be found in a separate paper (Walker and Ireland 1995). We note that microlensing has already been proposed as the source of observed variability in one sample of quasars (Hawkins 1993).

\section{Technique}

The observational approach to this experiment is essentially the same as is currently used for the Galactic microlensing experiments, differing mainly in the smaller number of sources which need to be monitored $\left(\sim 10^{2}\right.$ vs. $\sim 10^{6}$ ). (For an instrument like the MACHO camera observing a rich cluster at redshifts $0.01<z<0.05$, the mean optical depth to cluster microlensing is $\sim 10^{-2}$ if all the cluster dark matter is in compact form.) One significant extra requirement is that the target sources need to be identified as 
such. This can be done spectroscopically, using the 400 -fiber 2 degree Field instrument on the Anglo-Australian Telescope to isolate the quasars from the faint stars.

For sensible time-scales - between 20 minutes and a few years - the suggested monitoring experiment would be sensitive to dark matter lumps in the mass range $10^{-10}<M / M_{\odot}<1$. However, quasars are believed to emit their optical continuum from a region of dimension $\sim 10^{14} \mathrm{~cm}$ (e.g. Rees 1984), and the magnification would therefore be very small for masses less than $\sim 10^{-4} M_{\odot}$ where the Einstein ring is smaller than the apparent size of the source. Clearly the experiment is still sensitive to an interesting mass range, including as it does very low mass stars and substellar objects. Weekly monitoring over a period of a few years is sufficient to cover the mass range $10^{-4}<M / M_{\odot}<1$.

\section{Difficulties}

The principal difficulty with the proposed experiment lies in the recognition of microlensing-induced variability. Quasars are variable sources and one needs some means of distinguishing intrinsic variability from microlensing. In the case of Galactic microlensing one usually requires, for example, that an event be achromatic and time symmetric in order for it to be considered a possible lensing event; whereas the anticipated source structure for quasars suggests that asymmetric and chromatic events are quite plausible. In our favor is the observation that typical quasars do not vary profoundly (around $0.2 \mathrm{mag}$ ) in the optical band over a decade (Hook, McMahon, Boyle and Irwin 1994). Also in our favor is the fact that, because the optical depth to microlensing is much less than unity, any microlensing should show-up as distinct events on a relatively steady baseline.

These signatures are, however, insufficient as proof of a microlensing origin for any observed variability. Fortunately there is a unique property which cannot be mimicked by intrinsic variability: because the surface density of dark matter varies through the cluster, the microlensing event rate will similarly vary so that the process can, on a statistical basis, be verified as the variability mechanism.

\section{References}

Hawkins, M.R.S., 1993, Nature, 366, 242

Hook, I.M., McMahon, R.G., Boyle, B.J. \& Irwin, M.J., 1994, MNRAS, 268, 305

Paczyński, B., 1986, ApJ, 304, 1

Rees, M.J., 1984, ARA\&A, 22, 471

Udalski, A., Szymański, M., Katużny, J., Kubiak, M., Mateo, M., \& Krzemińskii, W., 1994, ApJL, 426, L69

Walker, M.A. \& Ireland, P.M., 1995, MNRAS, in press 